\title{
Molecular Electronics: From Physics to Computing
}

\author{
Yongqiang Xue ${ }^{1, *}$ and Mark A. Ratner ${ }^{2}$ \\ ${ }^{1}$ College of Nanoscale Science and Engineering, University at \\ Albany-State University of New York, Albany, New York 12203, USA \\ ${ }^{2}$ Department of Chemistry and Materials Research Center, \\ Northwestern University, Evanston, Illinois 60208, USA \\ To Appear in Springer Series in Natural Computing
}

Even if Moore's Law continues to hold, it will take about 250 years to fill the performance gap between present-day computer and the ultimate computer determined from the laws of physics alone. Information processing technology in the post-CMOS era will likely consist of a heterogeneous set of novel device technologies that span a broad range of materials, operational principles, data representations, logic systems and architectures. Molecular nanostructures promise to occupy a prominent role in any attempt to extend chargebased device technology beyond the projected limits of CMOS scaling. We discuss the potentials and challenges of molecular electronics and identify some fundamental knowledge gaps that need to be addressed for a successful introduction of molecule-enabled computing technology.

\section{INTRODUCTION}

The first functioning transistor was invented by Bardeen, Brattain, and Shockley in the late $40 \mathrm{~s}^{1.2}$. It is a bipolar junction transistor (BJT) made from a small block of germanium. The first integrated circuit (IC) was invented in 1961, which combined monolithic bipolar junction transistor and passive components on a single chip. This event marked the start of the microelectronics revolution ${ }^{3}$. The realization of an older transistor principle - the field-effect transistor (FET) - came about with the metal-oxide-silicon (MOS) transistor in 1962. After the development of the complementary MOS (CMOS) circuits, silicon-based MOSFETs nearly completely dominated digital logic circuits due to the ease of very-large-scale-integration (VLSI) and low power consumption ${ }^{4}$. For almost 40 years, the VLSI industry has followed a steady path of constantly shrinking device geometries and increasing chip size, resulting in a history of new technology generation every two to three years, commonly refered to as "Moore's Law". The 2004 International Technology Roadmap for Semiconductors (ITRS) now extends this device scaling and increased functionality scenario to the 22-nm technology node at year 2016, with projected minimum feature sizes below 10 nanometers and chips with more than 6 billion transistors ${ }^{5}$.

Equally remarkable with the CMOS scaling is the fact that most of these developments have been achieved with the same basic switching element (MOSFET), the same basic circuit topology (CMOS), and with a limited number of materials (up to about 15 elements in the 1990s). In many respects, progress in these areas has been straightford following the design scaling rules in the sense that no fundamentally new inventions have been needed ${ }^{6.7}$. However, there is no particular reason why Moore's Law should continue to hold: it is a law of human ingenuity, not of nature. Indeed, the current ITRS roadmap predicts that the scaling of the conventional CMOS technology will slow down or stop beyond the 22-nm node. Prior to that time, there are a large number of difficult technological challenges at the materials, device, circuit and system levels that must be met and overcome, many of which currently have no known solutions 5.8.9.10. This is because nanometersize MOSFET are no longer scaled short-channel devices with long-channel behavior. They are true nano-scale devices involving the creation and manufacture of objects within the regime of nanotechnology 11 .

In contrast to the CMOS scaling, the technological challenges for the information processing industry in the post CMOS era are quite different because it is far from clear what needs to be done ${ }^{12.13}$. Nanostructures exhibit a variety of interesting physical, chemical and biological properties, many of which can be significantly modified by the processing and environmental conditions and are not yet fully understood. Progress in innovative device design thus often has to come hand in hand with progress in fundamental knowledge of the physics and chemistry of nanostructures. Although there is a growing consensus that the near term extension of chargebased device technology will require a nanodevice technology that is architecturally compatible with CMOS and functionally supplementing rather than replacing CMOS, questions remain regarding the best direction to pursue for such nanoelectronics ${ }^{14}$. For example, what are the best functional nanostructures, carbon nanotubes, silicon/compound nanowires, or molecules/polymers? And what are the best device concepts, field-effect transistors, single-electron transistors, quantum-effect devices (resonant tunneling, quantum interference,...), etc? In the longer term, a new nanodevice technology may need to exploit electron and electronic charge/current in fundamentally new ways that are closely linked to the use of alternate state variables for representing information.

In general, nanostructured systems may span a broad range of materials, data representations, operational principles, and may function in different architectures and on different applications. Independent of the technology route that post-CMOS devices take, the operation 
and performance of nanometer-size devices will increasingly be governed by atomic-level variations in the materials/device structures and processing/environmental conditions. The prospect of device design through the bottom-up atom-engineering route of nanotechnology has far-reaching impacts on post-CMOS information processing technology either as monolithic systems or as polylithic hybrid systems interfacing to the scaled CMOS. Molecular nanostructures occupy a prominent role in any attempt to offer significant expansion in device functionality beyond the end of CMOS scaling.

In this work, we discuss opportunities for information processing based on quantum engineering of the physical states of molecules. Here we define a molecule broadly as a unit whose physical (electrical, magnetic, mechanical, optical...) and/or chemical (reactivity, solubility, molecular recognition...) properties are sensitive to atomic-scale modification of its structure and/or environment. Note that such a definition of molecule essentially covers all nanostructures as defined in the US National Nanotechnology Initiative (NNI) 15 , including atoms, organic molecules, polymers, nanotubes, nanowires, and nanoparticles, etc. Since the only practical generalpurpose information processing technology (besides human brain) currently available is based on silicon devices which use electron and electronic charge/current to drive electronic circuits performing Boolean logic, we limit our scope here to charge-based device technology. We don't consider device technology options that use fundamentally different physical representations of computational state such as optical computing and DNA computing. We will not discuss molecular spintronics either which forms a separate avenue of research, even though spin is intrinsically associated with electrons and nuclei.

Before we proceed to the main sections of this paper, we want to emphasize that our goal here is not to provide a complete survey of molecular electronics approaches that have been studied or proposed so far, but rather to identify the critial research needs in fundamental science that must be addressed in order to extend charge-based device technology through the molecular/nano- engineering route. Since any attempt in investigating the potentials of the nanotechnology route to information processing should be gauged in reference to both its ultimate physical limit and the limits of the ultimately scaled CMOS devices, we start with discussions of the ultimate physical limits to computation and the physical factors that account for the success of semiconductor technology and their limits in sections II and III respectively. We discuss the main topic of the paper, molecular electronics, in section IV. We conclude and summarize in section $\mathrm{V}$.

\section{ULTIMATE PHYSICAL LIMITS TO COMPUTATION}

Computers are physical systems, and the laws of physics dictate what they can and cannot do 16.17 . Much of the current activity in molecular electronics has been motivated by Feynman's pioneering work on the physical limits of miniaturization and computation ${ }^{18,19}$. Therefore we start the investigation of molecular electronics with examining the limits that the laws of physics place on the power of computers. There exists a vast literature on this topic ${ }^{20,21}$. Our discussion here follows that of Lloyd ${ }^{22}$, which explores the ultimate physical limits to the computational capacity of a computer with a mass of $1 \mathrm{~kg}$ occupying a volume of 1 litre (the so-called ultimate laptop computer) as determined by the speed of light $c$, the quantum scale $\hbar$ and the thermodynamic scale $k_{B}$.

\section{A. Speed Limits}

A digital computer performs computation by representing information in terms of binary digits or bits with logical states $|0\rangle$ and $|1\rangle$, and then processes that information by performing simple logical operations. Any boolean function can be constructed by repeated application of AND, NOT and FANOUT, which forms a universal set ${ }^{19}$. During such logical operations, the bits on which the operation is performed go from one state to another. The maximum speed per logic operation can thus be determined by how fast a quantum system can move from one distinguishable state to another, i.e., the maximum speed of dynamical evolution. Since the quantum measure of distinguishable states is the orthogonality of states involved, this is best illustrated by considering the minimum time needed for the NOT operation, which changes the $|0\rangle$ state to its orthogonal $|1\rangle$ state or vice cersa. This question is closely related to the AharonovBohm interpretation of the time-energy Heisenberg uncertainty principle $\Delta E \Delta t \geq \hbar^{23.24}$ : It is not that it takes time $\Delta t$ to measure the energy of a quantum system to an accuracy of $\Delta E$, but rather that a quantum system with spread in energy $\Delta E$ takes time at least $\Delta t=h / 4 \Delta E$ to evolve to an orthogonal state ${ }^{25}$. Instead of expressing the speed of dynamical evolution in terms of the standard deviation of energy $\Delta E$, Margolus and Levitin ${ }^{26}$ generalized the result to show that a quantum system with average energy $\mathrm{E}$ (relevant to its ground state energy) takes time at least $\Delta t=h / 4 E$ to evolve to an orthogonal state.

Since the simple logical operations of AND, NOT and FANOUT can all be enacted in the so-called controlled-controlled-NOT operation ${ }^{28}$, by embedding the controlled-controlled-NOT gate in a quantum context it is easy to show that the maximum speed of logic operation is limited by the energy input to the logic gate performing the operation as $4 E / h$. More complicated logic operations may involve system evolution cycling 
through a large number of quantum states. For evolutions that pass through an exact cycle of $\mathrm{N}$ mutually orthogonal states at a constant rate, it has been shown that the transition time between the orthogonal states is $\Delta t \geq \frac{N-1}{N} h / 2 E$, or the long-sequence asymptotic transition time is twice as long as it is for oscillation between $N=2$ states $^{26}$. Applying this result to a $1-\mathrm{kg}$ computer with energy $E=m c^{2}$ shows that the ultimate laptop can perform a maximum of $4 m c^{2} / h \approx 5.426 \times 10^{50}$ operations per second 22 .

\section{B. Memory Limits}

A system with $N$ accessible states can register $\log _{2} N$ bits of information, so the amount of information that can be registered by a physical system is related to its thermodynamoc entropy by $I=S(E, V) / k_{B} \ln 2$, where $S(E, V)$ is the thermodynamic entropy of a system with expectation value for energy $E$ confined to a volume $V$. When it is using all its memory space, the ultimate laptop can perform a maximum number of operations per bit per second of $\frac{4 E}{h} / \frac{S}{k_{B} \ln 2} \propto k_{B} T / \hbar$, where $T=\left(\frac{\partial S}{\partial E}\right)^{-1}$ is the operating temperature of the ultimate laptop in the maximum entropy state. A simple estimate of the maximum entropy for the 1-kg computer in a litre volume can be obtained by modeling the volume occupied by the computer as a collection of modes of elementary particles with total average energy $E$, and the maximum entropy $S(E, V)$ is that obtained by calculating the canonical ensemble over the modes which maximizes $S$ for fixed energy $E$ confined in a fixed volume $V$ with no constraint on the spread in energy $\Delta E^{29}$. Note that this is different from the canonical ensemble normally used for open systems that interact with a thermal bath at temperature T. Consequently the temperature $T=\left(\frac{\partial S}{\partial E}\right)^{-1}$ has a different role in the context of calculating the maximum entropy of a closed quantum system than it does in the case of an ordinary thermodynamic system interacting with a thermal bath.

At a particular temperature $T$, the entropy is dominated by the contributions from particles with mass less than $k_{B} T / 2 c^{2}$. The particles contribute energy $E=r \pi^{2} V\left(k_{B} T\right)^{4} / 30 \hbar^{3} c^{3}$ and entropy $S=$ $2 r \pi^{2} V\left(k_{B} T\right)^{3} / 45 \hbar^{3} c^{3}=4 E / 3 T$, where $r$ is the number of particles/antiparticles in the species multiplied by the number of polarizations multiplied by a degeneracy factor reflecting particle statistics ${ }^{29}$. A simple lower bound on the entropy can be obtained by assuming the energy and entropy are dominated by black-body radiation of photons, for which case $r=2$ (A recent derivation finds the same ultimate limits for information encoded using both matter and massless fields $\left.{ }^{30}\right)$. For a $1-\mathrm{kg}$ computer confined to 1-litre volume, the maximum entropy state corresponds to the operating temperature of $k_{B} T=8.1 \times 10^{-15} \mathrm{~J}, T=5.87 \times 10^{8} \mathrm{~K}$. The maximum entropy is $S=2.04 \times 10^{8} \mathrm{~J} / \mathrm{K}$, which corresponds to an amount of memory space of $I=S / k_{B} \ln 2=2.13 \times 10^{31}$ bits. When the ultimate laptop is using all its memory space, it can perform $4 \ln (2) k_{B} E / S \approx 10^{19}$ operations per bit per second 22 .

\section{Thermodynamics of Computation}

The role of thermodynamics in computation is made clear in the intimate link between information and entropy. Ordinary electronic computers are thermodynamic engines that do work and generate waste heat. Reducing the supply power and removing the heat produced have been main technology drivers throughout the history of computing. However, contrary to the intuitive thinking, Bennett showed in his pioneering paper ${ }^{31}$ in 1973 that it is possible to construct a general purpose computer using only reversible, i.e, one-to-one logical operations, therefore allowing in principle dissipation-less computing if we are willing to compute slowly. Energy is dissipated only when information is discarded. Landauer showed that irreversible, many-to-one operations such as AND or ERASE require heat dissipation of at least $k_{B} T \ln 2$ for each bit of information lost ${ }^{32.33}$. A closely related but separate energy disspiation limit has been established for communicating information. Again, in the absence of noise, i.e., interaction between the physical system carrying information and another uncontrolled physical system, the energy required for transmission of a unit of information can be made arbitrarily small if we are willing to do it slowly ${ }^{34}$. But, as shown by Levitin, a minimum energy of $k T$ must inevitably dissipate in order to transmit a unit of information over a noisy channel as a result of the interaction with uncontrolled degrees of freedom (environment) ${ }^{35}$. More recently, similar fundamental limits on the energy transfer associated with a binary switching transition have been derived in the context of semiconductor technology by Meindl and Davis 36 .

Besides these fundamental energy dissipation requirements, a realistic computer will inevitably be subject to errors during its operation. Error-correcting codes can be used to detect these errors and reject them to the emvironment at the dissipative cost of at least $k_{B} T \ln 2$ per bit. Typically such error-correcting operations must be done at a high rate in order to maintain reliable operation $^{22,32,33}$. The thermal load of correcting large numbers of errors alone can dictate the necessity of operating at a slower speed than the maximum allowed by the laws of physics 17,22 .

\section{LIMITS OF SEMICONDUCTOR TECHNOLOGY}

The discussion of ultimate physical limits to computation does not imply that we can construct a computer that operates at those limits. For example, it is inconceivable for present-day technology to control computers 
operating at $T=5.87 \times 10^{8} \mathrm{~K}$, or close to the temperature at which electrons and positrons can be produced thermally. Processing, storing and transmission of information requires that it be represented as the value of some physical quantitity, and physical laws control the materials and devices that are used to manipulate information ${ }^{37.38}$. Contemporary electronic computers operate at speed, memory and energy dissipation capabilities far lower than those dictated by the consideration of physical laws alone. From the physical perspective, such computers operate in a highly redundant fashion. However, there are good technological reasons for such redundancy.

\section{A. What Makes a Good Computing Device}

Many ingenious proposals for better computing devices were put forward and have been the focus of well-funded development efforts as silicon microelectronics continued its relentless drive toward miniaturization in the past four decades. But the only general-purpose digital computers that have ever been built were built with (in the chronological order) electrical relays, vacuum tubes, bipolar junction transistors and field-effect transistors. So why do so many ingenious schemes fail to realize their promise in electronic computation? The answer lies in the vast difference between the conditions in which devices are first discovered and demonstrated in the laboratories and those in a large system of many devices 39,40 .

For laboratory demonstration of a simple logic circuit, one needs only to choose a few proven devices and fine-tune their operating conditions as necessary to make them work well to perform a logic operation. But a large computer that contains tens of thousands to many millions of devices works in much less benign conditions. The output of one device is readily input to another, and so on through thousands of step or more. A large amount of communication among the many devices is entailed. There are frequent opportunities for a signal to be altered in its passage from one device to another, suffering attenuation, diffraction, dispersion and cross-talk on the path. The multiple physical and chemical processes used in mass-production of the large numbers of component lead to small differences in device characteristics. In addition, chemical reactions and diffusion lead to additional unpredictable changes in devices over time adding to the uncertainty inherent in manufacturing.

While the net result of the hazard factors is tolerable in a single logic operation, information must pass sequentially through a large number of stages in the computing system. Information would soon be lost if the errors introduced were allowed to propagate and accumulate from stage to stage. Digital representation of information can prevent this by resetting the output of a device to one of the standard values after each step. The output of a device may be required as input by other devices. The transmission of a signal to a multiplicity of destinations is known as fan-out and devices for computers must be

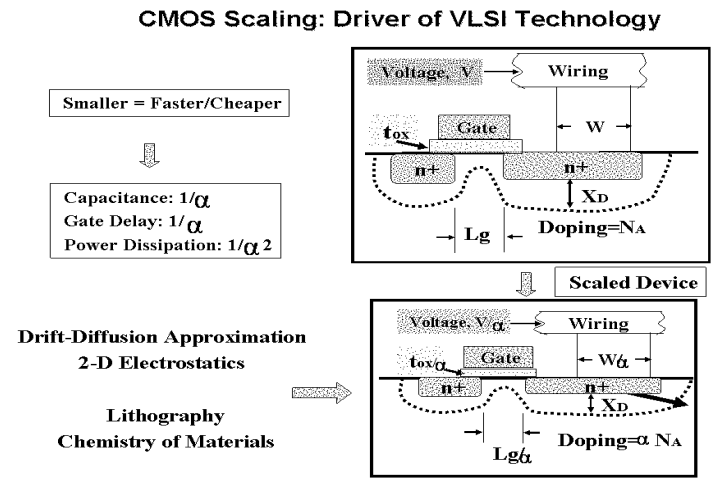

FIG. 1: Schematic illustration of MOSFET scaling

able to provide fan-out. The standarization of signals and fan-out require that an electrical device controls voltage and current larger than those needed to operate it, or a device should have both current and voltage gains. Gain is essential to digital devices in order that the switching transition at the threshold occupies a small part of the signal swing and allows high noise margin. In addition, it is desired that a computation in a machine proceed in one direction, from input to final results. Each device should operate only on its inputs and not be sensitive to the actions or status of the receipients of its outputs. This property is known as input-output (I/O) isolation and is required in computer devices.

The need for I/O isolation, fan-out and high gains put a severe limitation on the choice of devices suitable for large computing systems, which was only satisfied by the electrical relays, vacuum tubes and transistors. Careful examination of other proposed devices showed that they have difficulty in satisfying the three conditions simultaneously 39.40 . The transistors, especially the silicon MOSFETs, eventually win out due to their small size, fast speed, operating stability and low-power consumption.

The rest of this section is thus devoted to the challenges and limits facing semiconductor technology toward the end of the ITRS roadmap as shaped by the laws of physics. Such limits can be codified at a hierarchy of levels of materials, devices, circuits and systems ${ }^{8}$. Many review papers have been written on various limits to silicon technology $y^{8,9,10,41,42,43}$. We'll focus our discussion here only on those aspects of the materials and device limits of silicon technology that are likely to be relevant to the CMOS-like route to nanoelectronics through molecular/nano- engineering.

\section{B. Materials Limits of MOSFET Technology}

Materials limits are determined by the properties of the particular semiconductor, dielectric, and metallic materials used but are essentially independent of the structural features and dimensions of particular devices. There are 
three key materials limits: gate stack including both gate dielectric and gate electrode, doping in silicon, and contact formation 41 .

The gate insulator in a MOSFET needs to be thin compared to the device channel length (a few percent) in order for the gate to exert dominant control over the channel potential. But quantum mechanical tunneling of carriers through the insulator increases exponentially with decreasing insulator thickness. This puts the limit of silicon dioxide $\left(\mathrm{SiO}_{2}\right)$ thickness near $1.0 \mathrm{~nm}$ or five atomic layers thick for sub-20 nm MOSFET operating at $1 \mathrm{~V}$ in order to accomodate standby power requirments in most IC applications ${ }^{44}$. In addition, not all the applied gate voltage is efficiently coupled to the channel due to the polysilicon depletion effects in the gate electrode and quantum confinement effects in the silicon substrate, which add aproximately $0.8 \mathrm{~nm}$ to the equivalent electrical thickness in the gate capacitor 41 . One solution is to introduce high- $\kappa$ dielectric material which reduces the tunneling current while maintaining strong gate electrostatic control. But as the dielectric constant of the insulator increases, the band gap tends to decrease and the band lineup at the silicon-dielectric interface can be quite asymmetric. To avoid thermal emission over a Schottky barrier, a barrier height of more than $1 \mathrm{~V}$ is needed for both electron and hole. Another major barrier any new dielectric material will have to overcome is to achieve almost the same low-defect density as that of the native $\mathrm{Si}-\mathrm{SiO}_{2}$ interface. This puts significant constraint on the choice of dielectrics and their processing steps. In addition, many of the new dielectric materials are unstable in direct contact with silicon and also in the presence of the polysilicon gate. Thus it is likely that the entire gate stack will have to be replaced with metal gates replacing polysilicon, which has the advantage of lifting the gate depletion effect. But polysilicon has the advantage that it can be doped either p-type or n-type, shifting the workfunctions so that it is suitable for both NMOS and PMOS devices. In contrast, two different gate metals are needed for incorporation into a CMOS flow with workfunctions near the conduction and valence band edges respectively, which complicates enormously the fabrication process 41 .

The second issue is associated with the need for ultrashallow source/drain junctions to reduce the parasitic resistance of the source/drain extension regions and the short-channel effect due to drain electric field extending through the channel region. This requires increasing the doping density of the source/drain region while maintaining abrupt doping profile across the silicon body. However, the maximum dopant concentration that can be dissolved in silicon under equilibrium conditions (the solid solubility) is $\approx 2 \times 10^{21}$ atoms $/ \mathrm{cm}^{3}$ ) (achievable for arsenic at $\left.\approx 1200^{\circ} C\right)^{45}$. Although transient laser annealing can introduce arsenic in metastable electrically active concentrations near or above the solubility limit, there is an enormous driving force that tends to deactivate the arsenic during any subsequent thermal cycling 46 . The dominant technology used for doping silicon is ion implantation, which provides precise control of the placement and quantity of doping atoms. But the implantation process produces considerable damage in the silicon substrate as a result of the nuclear collisons involved in the stopping process. Dopants diffuse by interaction with point defects in the subsequent thermal anneal to achieve the desired doping profile. The mechanisms underlying the defect formation and dopant diffusion process are far from being fully understood 47 .

The third issue is associated with the junction contact formation. Contacts in silicon technology are normally made with self-aligned silicides containing heavily doped silicon. This process provides an ohmic contact covering the area of the source/drain diffusion and minimizes the contact resistance. Further reducing the contact resistance with decrease of feature size requires increasing the silicon doping and reducing the Schottky barrier height. The doping is limited by the solid solubility as discussed earlier. Barrier height engineering in metal-silicon system remains not fully understood despite its obvious technical importance. In addition, the silicide formation process consumes the top portion of silicon as the metal is deposited and reacted to form the silicide, this can increase sheet resistance of the source/drain extension region and also change the dopant structure adjacent to the metal.

\section{Device Limit of MOSFET Technology}

Historically MOSFET scaling has been governed by the need to preserve the good electrostatic behavior at the reduced device dimension, i.e., reducing supply voltage and gate insulator thickness and increasing doping concentration. The traditional limit of device scaling is determined thus by the effects that modify the ideal electrostatic contol. These include quantum effects due to tunneling leakage through gate insulator, tunneling through body-to-drain junction, direct source-to-drain tunneling, thermal effects due to thermally generated subthreshold current at room temperature and also the increasing sensitivity to minute fabrication spreads. In addition to such limits intrinsic to small device size, other limits more intimately connected to the materials and device structure of the ultimately scaled MOSFET have been proposed which, as accutely pointed out by Fischetti, suggests changing the "nature" of the nanometersize MOSFETs moving toward the sub-10nm regime ${ }^{43}$.

The most fundamental one seems to be that set by the long-range Coulomb interaction between the channel electron and the "high-density" electron gas in the highly doped source, drain and gate electrodes. This is reflected in: (1) the emission and absorption of the lowfrequency plasmon (on the order of magnitude of $m e V$ ) in the source/drain by the channel electrons which thermalizes the hot-electron distribution in the channel and indirectly reduces the effective electron velocity; (2) the "Coulomb" drag between the channel electron and electrons in the gate (also plasmon-mediated) across the very 
thin insulator results in a direct loss of momentum of the channel electrons. Both effects may contribute to the breakdown of "ballistic" transport widely assumed in current theoretical estimates of the MOSFET scaling limit 48: Short channel is required for "ballistic" transport, but the increased strength of Coulomb interaction may kill it at the outset $\frac{43}{3}$. Combining with other less fundamental but equally important effects such as "remote" phonon scattering in the gate stack and scattering accompanied with substrate engineering, this may contribute further to the end-of-the-road scaling scenario that there may not be a single end point for scaling, but instead many end points, each adapting optimally to its particular applications ${ }^{42}$.

\section{MOLECULAR ELECTRONICS: FROM PHYSICS TO COMPUTING}

\section{A. Motivation and Definition}

Even if Moore's Law continues to hold, it will take about 250 years of exponential scaling to fill the gap between the ultimate laptop operating on $10^{31}$ bits at $10^{51}$ operations per second and the present-day laptop operating on $10^{11}$ bits at $10^{10}$ operations per second. Although the ultimate laptop operates at conditions that do not seem to be contollable at all from present-day technology, new physical principles may be imagined that turn today's inconceivable into tomorrow's common sense if we remember quantum physics has only 100 years' history. But we are not concerned with such exotic possibilities beyond the horizon of current understanding of physical laws. The technological goal of molecular electronics is instead to extend the performance increase of chargebased device technology beyond that perceivable from CMOS scaling at the projected end of ITRS roadmap as far as possible, based on innovative utilization of functional nanostructures and quantum mechanical laws.

Although many technological barriers exist for which there are currently no known solutions, the past success of CMOS scaling gives us all reason to believe that the projected goal of the CMOS scaling at 2016 will be sur$\operatorname{passed}^{49}$, at which point the ultimate MOSFET will have gate oxide thickness in the 1.0-nm range, channel thickness in the 3.0-nm range, and channel length in the 9.0$\mathrm{nm}$ range $\mathrm{e}^{50}$. Since CMOS technology is the only practical general-purpose information processing technology (besides human brain) currently available, investigation along the molecular electronics route should be gauged in close reference to the continually scaled CMOS devices in both its conventional and "nonclassical" forms 51.52 . In addition, the future devices and their target performance metrics should meet the generic criteria of: (1) that they need to be of high performance in terms of speed and density while remaining energy efficient; (2) that they should be structurally stable under room temperature operation and not be dominated by parametric variations due to processing and environmental conditions; (3) that they should be scalable through multiple generations with integer multiples of performance. In the near term, they might preferably be capable of integration on a CMOS platform, but the long term options should be kept open (remember the 250-year span!). Consequently we shall consider materials and device issues associated with both molecular/nano- engineered devices that are structurally and/or functionally similar to CMOS devices (referred to as CMOS route hereafter) and molecular/nano- engineered devices that are configured for information acquisition, sensing, storage and transmission in ways fundamentally different from the CMOS devices (referred to as Non-CMOS route hereafter).

In the preface to the first edition of his widely popular textbook on semiconductor devices published in 1969, Sze defined a semiconductor device as a unit which consists, partially or wholly, of semiconducting materials and can perform useful functions in electronic apparatus and solid-state research 53 . Correspondingly we define a molecular electronic device as a system which consists, partially or wholly, of individual molecules and can perform useful functions in electronic apparatus and nanostructure research through atomic-scale control. We only discuss molecular electronics for applications in information processing device here and leave the discussion of molecular electronics as "artificial" laboratory of nanoscopic physics for other efforts $\$ 54.55$.

\section{B. Molecular Electronics: CMOS Routes}

\section{Molecular Transistor}

Three-terminal devices, i.e., transistors, have been indispensable for building digital logic systems based on semiconductor technology due to the stringent requirement of I/O isolation, large noise margin and signal gain. Molecular field-effect transistors (MolFET), where the active part of the device is composed of quasione-dimensional (Q-1D) nanostructures like carbon nanotubes or nanowires, have been widely studied that are structurally and functionally similar to their CMOS ana$\log 56.57 .58 .59 .60$. Q-1D nanostructures offer additional advantage as alternative channel materials in the CMOS route since they can function both as active devices and interconnects and thus have the potential to provide simultaneously two of the most critical functions in any integrated nanoelectronics $\frac{61,62,63}{}$. Experimental progress on single devices has been fast, and useful simple circuits like inverter, mixer and decoder have been demonstrated $64.65,67.68,69,70$. There are many points of confluence between the technologies of the scaled silicon devices and Q-1D nanostructured junctions and transistors, including the integration of high- $\kappa$ gate stack, homo(pn)and hetero- junction diodes and transistors, substrate engineering (strain) and "non-classical" transistor structures 62.63 .71 .72 . Investigation along this route provides thus an ideal reference point both for exploring novel de- 
From Semiconductor Microelectronics to Molecular Nanoelectronics: Materials, Physics, and Devices

(a)

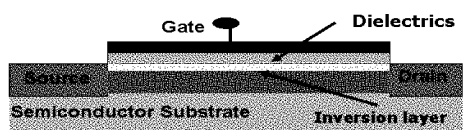

(b)

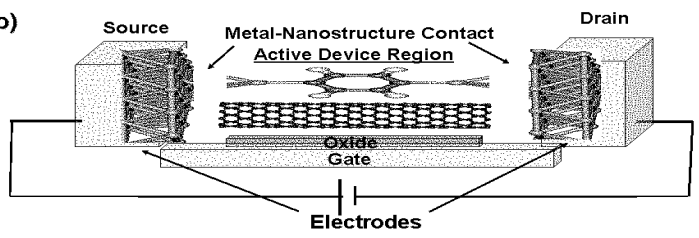

FIG. 2: Schematic illustration of the device structures of a conventional CMOS device and a typical nanodevice.

vice design at the molecular scale and for re-examining the physical principles of semiconductor microelectronics from the bottom-up approach. Here carbon nanotube and semiconductor nanowire offer subtle but significant differences in their prospect for post-CMOS information processing.

Carbon nanotubes (CNT) are hollow cylinders composed of one or more concentric layers of carbon atoms in a honeycomb lattice arrangement, which typically have a diameter of 1-10 $\mathrm{nm}$ and a length of several nanometers to several micrometers. In addition to the small size, CNTs offer some salient features that make them attractive candidates for electronic devices 57 : (1) The quasi-1D structure implies a reduced phase space for carrier scattering by both impurity and lattice vibration. It also leads to distinctly different electrostatic behavior from the planar silicon device which affects both screening and tunneling. (2) The C-C $s p^{2}$ bonding leaves no dangling bond on the surface. In particular, for single-wall carbon nanotube (SWNT) all carbon atoms are surface atoms. CNT electronics are not bound to use $\mathrm{SiO}_{2}$ as an insulator and novel transistor structures like surrounding gate transistors can be adapted. (3) The strong C-C $s p^{2}$ bonding gives CNTs high mechanical and thermal stability. Current densities $\geq 10^{9} \mathrm{~A} / \mathrm{cm}^{2}$ can be sustained. Several critical issues related to contact, doping and scattering remain to be sorted out for further development of CNT-based nanoelectronics.

In contrast to silicon MOSFET, the source, drain and gate electrodes in MolFET are currently made from deposited or lithographically defined metals. The Schottky barriers at the CNT-metal contacts play a significant role in determining the transport characteristics 57.73 .74 (we can also expect that the Schottky barrier problem will play an increasingly important role as MOSFET scales toward sub-10nm regime, since the low-frequency plasmon in the doped source/drain region can be removed by using metal electrodes). Due to the Q-1D geometry, both the barrier height and barrier shape are important in determining the relative importance of tunneling and thermionic emission across the barrier. The recent ob- servation of ohmic contact using Pd provides a particular challenge $\mathrm{f1.72}^{7 \mathrm{7}}$ as the previous theoretical study shows similar Schottky barrier for Pd and Au that have similar work functions. However, the model used assumes only electronic coupling across the interface with fixed atomic structure. Transition metals including both $\mathrm{Ti}$ and $\mathrm{Pd}$ are known to be chemically active attaching to CNT surface and can form carbide immediately adjacent to the interface 56.57 Recent experiments have also shown that Schottky barriers can be significantly lowered by chemical treatment of the metal-CNT interface ${ }^{75}$. Work will be needed to extend the theoretical model for better study of the interface chemistry including structural relaxation effects in the configuration of CNTFET with different gate structures.

Doping in a semiconductor typically implies introducing a shallow impurity atom into the host lattice using ion implantation or thermal diffusion accompanied by creation of lattice defects $\frac{47}{}$. But it may take a fundamentally different approach in CNTs. For example, doping in carbon nanotubes can be introduced chemically by exposing the CNT surface to alkali metals, by inserting $C_{60}$ molecules inside the CNT, by surface functionalization with molecules/polymers for charge-transfer doping (which is essentially the electronic basis of sensing). In addition, the doping type can be converted between ptype and n-type by chemical treatment using e.g. oxygen and molecular hydrogen56.57. Doping in nanotubes can also be introduced physically using electrostatic gating or contact-induced charge transfer ${ }^{76}$. "Self-doping" mechanisms for intrinsic SWNT caused by curvature induced charge redistribution have also been proposed, which shift the Fermi-level position inside the band gap ${ }^{77}$. Despite its obvious importance, comprehensive experimental and theoretical study and a coherent physical picture of the various doping mechanisms, including both electronic and structural consequences, have not yet appeared. A particularly interesting question in this regard is the optimal doping limit in carbon nanotubes for both physical and chemical doping mechanisms.

The major scattering mechanisms in CNTFET are those due to defects including dopant, gate stack and phonon. Due to the reduced phase space, the probability of back-scattering by defects and acoustic phonon is significantly reduced at low-bias compared to the planar silicon devices 78.79 .80 . The absence of reactive dangling bond states at the CNT surface also make it less likely to suffer significant scattering due to the interface states and charge traps at the channel-gate interface. But it remains unclear how these favorable conditions may be modified at high-bias. These include optical phonon emission by the energetic carrier, the injection of carriers into the gate dielectric and the resulting gate insulator degradation, remote phonon scattering between channel electrons and gate phonons, and the structural stability adjacent to the intrinsic or doping induced defect site. The optical phonon scattering length has been estimated at $\approx 10$ $\mathrm{nm}^{79}$, but in the absence of a realistic quantum transport 
model of electron-phonon coupling in CNTFET, this result should be taken with reservation ${ }^{81}$. Many fundamental knowledge gaps need to be addressed before we can have a convincing picture of the performance limit of CNTFETs in comparison to that of the ultimately scaled MOSFET. The recent report on suspended carbon nanotubes seems to suggest a cleaner platform for investigating many of the issues involved ${ }^{82}$.

A different scenario applies to the nanowire FETs (NWFET), which seem to be less controversial. The vapor-liqiuid-solid phase growth process using nanoclustered catalyst pioneered by the Lieber group has led to the fabrication of single-crystal silicon nanowires 62 , where the size distribition of the nanowires is determined by that of the catalyst nanoclusters. Both ntype and p-type dopants can be selectively inserted during the nanowire growing process. This has opened up the scheme of fabricating complementary logic circuits on the single silicon nanowire, where source/drain electrodes can be lithographically defined after the doped segments have been grown. Since the diameter of the nanowires is typically several tens of nanometer, wellknown techniques in forming metallic contact in planar silicon device can be adapted leading to low barriers and low resistance contacts 62,63 . More recently, innovative techniques have been reported that solve the integrated contact and interconnect problem through selective transformation of silicon nanowires into metallic silicide nanowires 83 . The single-crystal metallic silicides have high conductivity and high failure current, while being capable of forming atomically sharp metalsemiconductor heterostructures with the silicon nanowire of similar diameters. This opened up the possibility of an ultra-dense integrated nanosystem that integrates both active device area and high-performance interconnect from a single nanowire building block while benefitting from the knowledge gained in the planar silicon devices (in particular the silicon-on-insulator approach) with minor modifactions. In addition, different elemental, binary and ternary nanowires can be fabricated using the same vapor-liquid-solid geowing process, providing a significant design freedom for system designers ${ }^{62.63}$.

Both carbon nanotube and nanowire field-effect transistors have been demonstrated showing favorbable performance compared with the state-of-the-art silicon MOSFET, while leaving substantial room for materials and device design optimization. Carbon nanotubes, even though of much smaller diameter than silicon nanowires, do not have the advantage of integrated metallic ocntact on the single-tube basis. This is because the reduced phase space and the correspondingly low electron density of states in the metallic SWNT do not allow rapid relaxation of carriers injected through the channel, which has to be connected to a larger area metal electrode to allow I/O separation and efficient heat removal. Athough this may be remedied by using bundles of metallic SWNT or metal nanowires, further materials and fabrication challenges need to be resolved in addition to the Schottky barrier problem in such interfaces. The challenge for nanowire FETs is instead to scale the nanowire to true molecular dimension while maintaining scalable performance gain ${ }^{84}$.

\section{Molecular Interface to CMOS}

Direct integration of molecular functionality with the scaled CMOS technology forms a starting point for hybrid top-down and bottom-up approaches. Such hybrid approaches may combine a level of advanced CMOS lithographical design pattern that represents designerdefined information and a level of molecular structures self-assembled with great precision and functional flexibility. This combines the advantages of nanoscale components, such as the reliability of CMOS circuits and the minuscle footprints of molecular devices, and the advantages of patterning techniques, such as the flexibility of traditional photolithography and the potential low cost of nanoimprinting and chemically directed self-assembly, to enable ultra-dense circuits with acceptable fabrication costs.

One promising direction is to use molecules as charge storage elements for nonvolatile memory in the MOSFET structure. Nanocrystal and quantum-dot memories are examples of flash memories that utilize quantum dots between the gate and the channel of the field effect transistor to store electrons, which screen the mobile charge in the channel, thus inducing a change in the threshold-voltage or conductivity of the underlying channe ${ }^{85.86 .87}$ The quantum dots are isolated from the gate, and their processing can be accomplished together with CMOS processing. Both metallic and semiconductor nanocrsytals embedded in the gate oxides have been explored, but to enable reliable operation utilizing the single-electron effect at room temperature, truly molecular dimension $(\approx 1 \mathrm{~nm})$ quantum dots are preferred.

Recent work has demonstrated the integration of fullerenes including $C_{60}$ and $C_{70}$ in the gate stack of CMOS technology 88,89 . An electrically erasable programmed read-only-memory (EEPROM) type device was fabricated by effecting molecular redox operations through non-volatile charge injection, which occurs at a specific potential of the fullerene molecules with respect to the conduction band of $\mathrm{Si}$ at the $\mathrm{Si} / \mathrm{SiO}_{2}$ interface. Compared to metal and semiconductor nanocrystals which have non-negligible size variations, the monodisperse nature and small size of fullerene molecules lead to large and accurate step-wise charging into the molecular orbitals and may potentially provide reliable mutilevel storage with electrostatic control.

Alternatively, the body thickness control in the quantum-dot memory can be solved using CNTFETs which have monodisperse nanoscale cross sections. A new nonvolatile memory structure has been reported which uses a back-gated CNTFET as sensing channel and metal nanocrystals embedded in the dielectric layer near the SWNT as charge storage media ${ }^{90}$. The gate electrode regulates the charging and discharging of the metal nanocrystal, which imposes a local potential change on 
the nanotube channel and alters its electrical conduction. The device shows clear single-electron sensitivity and Coulomb blockade charging ${ }^{90}$.

A closely related concept is to use redox-active molecules self-assembled on nanowire field-effect transistors for nonvolatile memory and programmable logic applications ${ }^{91}$. Multi-level molecular memory devices have been demonstrated using porphyrin molecules selfassembled on $\mathrm{In}_{2} \mathrm{O}_{3}$ nanowire transistors for nonvolatile data storage up to three bits per cell ${ }^{92.93}$. Charges were placed on the redox active molecule. Gate voltage pulses and current sensing were used for writing and reading operations. Here replacing the gate insulator layer with self-assembled molecular components reduces significantly the device size, which simplifies fabrication and may aviod potential damage to the molecular component during the gate stack formation. In addition, different molecule-nanowire combination may be chosen leaving enormous room for design optimization. This seems to be a very promising direction, although many fundamental questions regarding the nature of the molecular states during read and write operation remain to be sorted out.

\section{Molecular Electronics: Non-CMOS Routes}

\section{Molecular Switch}

The situation for designing three-terminal switching devices on the molecular scale becomes much less clear once we move out of the proven domain of CMOS-like information processing ${ }^{94}$. This is exemplified by the lack of field-effect transistor effect in devices made from short $(\approx 1 \mathrm{~nm})$ molecules, since effective gate control requires the placement of gate in close proximity to the molecule (a few angstrom away) while avoiding overlap with the source/drain electrodes ${ }^{96}$. One approach to demonstrate strong gate control in such small scale is to use an electrochemical gate by inserting the device in electrolytes. Here the gate voltage falls mostly across the electrical double layer at the electrode-electrolyte interface which is only a few ions thick, and strong field effects on the source/drain curent have been observed for a perylene tetracarboxylic diimide molecule $2.3 \mathrm{~nm}$ long covalently bonded to two gold electrodes at gate voltage of $-0.65 \mathrm{~V}$ due to the field-induced shift of molecular orbitals relative to the electrode Fermi level ${ }^{97}$. However, further increasing gate voltage causes the device to break down. The electrochemical gating techniques has also been applied to CNTFETs ${ }^{98}$, but the scaling characteristics of such electrochemical transistors remains unknown.

Another way of achieving a strong field regulation effect is to put charged species in close proximity to the molecules. One recent experiment demonstrated the modification of current-voltage characteristics through a single-molecule in a STM junction by nanometer-sized charge transfer complex, where the electron acceptor is covalently bonded to the junction molecule and the electron donor comes from the ambient fluid. The effect was attibuted to an interface dipole which shifts the Fermi level of the substrate relative to the molecular orbitals 99 . Another approach used scanning tunneling microscope (STM) contact to styrene-derived molecules grown on a Si(100) surface. The strong field effect arises from charged dangling bond states on the silicon surface, the electrostatic field of which shifts the molecular levels relative to the contact Fermi level. The effect can be modulated by STM manipulation of the surface charging state or the molecule-charged centre distance 100 .

Switching by mechanical movement of an atom in the molecule has been proposed for a long time. An ingenious purely mechanical computer has recently been demonstrated by researchers from IBM, which was made by creating a precise pattern of carbon monoxide molecules on a copper surface ${ }^{101}$. Tiny structures, termed "molecular cascade", have been designed and assembled by moving one molecule at a time using an ultra-high-vacuum low-temperature STM, that demonstrated fundamental digital logic OR and AND functions, data storage and retrieval, and the "wiring" necessary to connect them into functioning computing circuitry. The molecule cascade works because carbon monoxide molecules can be arranged on a copper surface in an energetically metastable configuration that can be triggered to cascade into a lower energy configuration, just as with toppling dominoes. The metastability is due to the weak repulsion between carbon monoxide molecules placed only one lattice spacing apart.

To overcome the intrinsically slow speed due to atomic/molecular motion, a molecular electromechanical switch has been proposed. An early suggestion of atomic relay transistor proposed to use the mechanical motion of an atom to cause conductance change or switching of an atomic wire ${ }^{102}$. Theoretical calculations suggest high switching speed of $\geq 30 \mathrm{THz}$ or $\geq 100$ Thz if a silicon or carbon atom is used as the switching atom respectively, where a displacement of the switching atom by only one diameter would change the conductance of the atomic wire by orders of magnitude $\frac{103,104}{}$. Such an atomic relay transistor was recently demonstrated using electrochemical gate control of silver atoms within an atomic-scale junction ${ }^{105}$. A switching time of less than $14 \mu S$ was estimated. An early molecular version of electro-mechanical amplifer was demonstrated using STM manipulation of $C_{60}$ molecules, where current flowing through the $C_{60}$ molecule can be modified exponentially upon minute compression of the molecule by the STM tip 106 . More recently, a molecular version of the atom relay transistor has been demonstrated based on the rotation of the di-butyl-phenyl leg in a Cu-tetra-3,5 di-tertiary-butyl-phenyl porphyrin molecule, where the intramolecular motion of the switched leg is controlled mechanically by the tip apex of a noncontact atomic force microscope $e^{107.108}$. The comparison of the experimental and computed forces shows that rotation of the switched leg requires an energy of less than $100 \times 10^{-21} \mathrm{~J}$, or four orders of magnitude lower than the state-of-the-art MOS- 
FET.

The three-terminal switching devices just discussed, although ingenious and scientifically provoking, do not seem to satisfy the requirements of $\mathrm{I} / \mathrm{O}$ separation, gain and fan out for digital applications and there is no known scheme for extending them to large scale integration. Several two-terminal molecular switching devices have been proposed and demonstrated based on the reversible conformational change upon application of an electrical field109.110.111.112.113.114. Different mechanisms have been proposed for such bistable molecular devices $112,113,114,115,116,117,118,119$. Other bistable devices showing negative differential resistance have also been observed ${ }^{120.121}$. The two-terminal bistable devices have a long history in solid state electronics including in particular tunneling and resonant tunneling diodes based on semiconductor homo- and hetero- junctions ${ }^{122}$. Despite the enormous efforts put into logic design using twoterminal devices, sucess is limited ${ }^{123}$. And it is now well known that the bistable characteristics is unfavorable for large computing system in many ways 39,40 . The critical point is that gain in the bistable logic depends on biasing the circuit close to the threshold so that the addition of only a small input can cause a large change in the output. This puts great demand on the precision with which this can be done and gain is hard to realize in a noisy world with variable components. In addition, there is no standardization of signal values and there is no convenient inversion operation. This has forced research innovations in molecular electronics architecture ${ }^{124.125}$. Similar objections apply to cellular automata type devices, for which molecules have been suggested for optimal implementation 39.40 . In the cellular automata approach, connecting devices together by wiring is avoided by letting each device interact directly with its nearest neighbours. Previous research suggests that the capabilities of cellular automata in large computing systems are limited: they do not allow efficient execution of frequent access to memory and branching to other computational routines because interactions with distant information occur by shifting data one step at a time. It is not clear yet how much advantage molecular self-assembly can bring to cellular automata or other collective computing paradigms ${ }^{126}$.

\section{Molecular Single-Electron Devices}

Single-electron devices - in which the addition or subtraction of a small number of electrons to very small conducting particles can be controlled at the single-electron level through the charging effect - have attracted much attention from the semiconductor industry as an alternative device technology that could replace CMOS beyond the $10-\mathrm{nm}$ frontier ${ }^{127,128,129}$. The previous discussion of molecular quantum dot memory has highlighted the potential advantage of molecular components in singleelectron memories. For logic applications, molecular implementation of single-electron transistors is equally important since molecular-scale field effect transistors cannot help solve the key problem of transistor parameter sensitivity to channel length. Research in the past decade shows that there are two major obstacles preventing the wide-spread application of single-electron logic: (1) the need to operate at very low temperature; and (2) the ultra sensitivity to background charge noise.

The potential size advantage of molecular components to enable room-termperature operation is obvious. Both theory and experiment show that for reliable operation of most digital single-electron devices, the single-electron addition energy $\left(E_{C}\right)$ should be approximately 100 times larger than $k T^{129}$. This means that for room temperature operation, $E_{C}$ should be as large as $3 \mathrm{eV}$, or quantumdot size about $1 \mathrm{~nm}$. Molecular electronics offers a solution to this scaling limit by taking advantage of the bottom-up self-assembling process. In addition, using molecules with precise chemical composition may potentially solve the reproducibility problem in conventional metal/semiconductor clusters or electrostatically defined quantum dots in two-dimensional-electron-gas (2DEG) due to the size and shape fluctuations. Note that singleelectron effects have also been demonstrated using carbon nanotubes, but their larger size makes them less likely candidate for reliable room temperature operation ${ }^{130,131}$. The solution of the random background charge problem is much more difficult. Note that the electrostatic potential associated with random charged impurities in the environment is a problem for any nanoscale devices. But it poses a particularly tough problem for single-electron devices because of their large charge sensitivity.

A comparison between the conventional approach and several representative single molecule-based singleelectron devices shows clearly the new physical processes introduced by the use of molecular-scale components $132,133,134,135,136,137$. The molecular-scale dimension of the quantum dot leads to two intrinsic effects due to the ultra-small size: (1) both the wave function and the energy of the discrete electron states of the quantum dot depend on the size, shape and net charging state of the quantum dot; (2) Due to finite number of degrees of freedom and lack of an efficient relaxation mechanism on the quantum dot, the quantum dot may stay in nonequilibrium state and self-heating may occur during the cycle of single-electron transfer. In addition, as electrons are added or removed from the molecular quantum dot, both the shape of the molecule and its position relative to the contacts may be altered. The electron state of the molecular-scale component is also sensitive to the atomicscale change of the environment, e.g. due to presence of surface states which in turn may be modified by surface adsorption, the presence of impurities on the contact surface and/or the interaction with neighbor quantum dots. Treatment of all the above processes goes beyond the conventional theory of single-electron tunneling and is important for quantitative and realistic evaluation of their figures of merit.

So far, these devices have been formed by techniques excluding practical fabrication of integrated circuits. But there are good prospects for chemical synthesis of special molecules that would combine the structure suitable for 


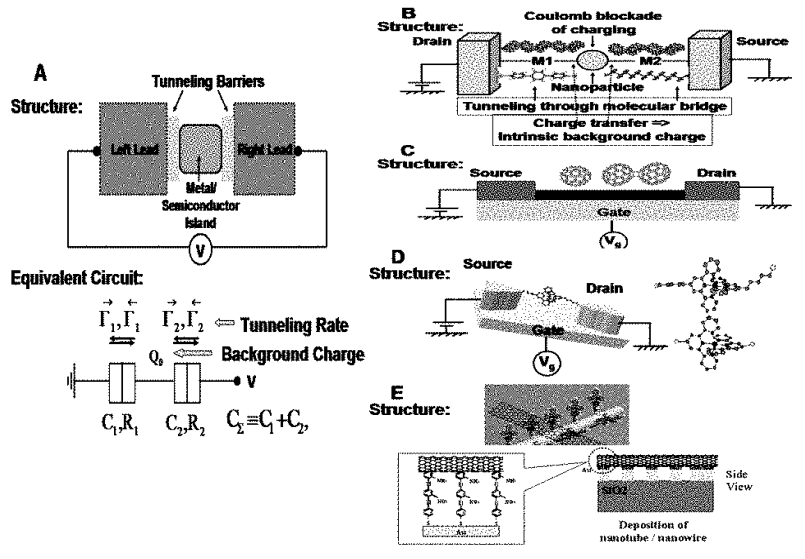

FIG. 3: A, Typical structure and equivalent circuit of the conventional single-electron devices. B, Self-assembled or biodirected assembly of single-electron device fabricated through synthetic routes. The nanoparticles are connected to the electrodes and/or to each other through either organic linkers or biomolecules with molecular-recognition capability ${ }^{132.138}$. C, A quantum dot is formed by a single C-60 or C-140 molecule physisorbed between two metal electrodes ${ }^{133.137}$. The molecule may start oscillating as discrete charges are added to or extracted from the molecules through the contact. D, The quantum dot is a single metal atom embedded within a larger molecule and connected to the metal contact pads through insulating tethers ${ }^{134,135}$. E, The molecule can also be adsorbed on top of a nanowire transistor which provides the source/sink of single electrons ${ }^{92}$.

single-electron tunneling with the ability to self-assemble from solution on prefabricated nanostructures with acceptable yield, opening a way to generically inexpensive fabrication of VLSI circuits. For logic circuits, the random background charge effects remain hard to overcome. Nevertheless, it has been suggested that the hybrid molecule-CMOS circuits, or "CMOL" circuits, that combine CMOS stack with molecular single-elctron devices interconnected by nanowires, in defect-tolerant architectures that allow to either tolerate or exclude bad devices, may become the basis for implementation of novel, massively parallel architectures for advanced information processing, e. g., self-evolving neuromorphic networks ${ }^{129}$. Such hybrid approach can help to solve the low gain of single-electron transistors, but it remains to demonstrate reliable high-performance digital circuits.

\section{Molecular Quantum-Effect Devices}

Intensive research on semiconductor heterostructures in the past three decades has generated many novel device concepts based on tunneling, resonant tunneling, real-space transfer, hot-electron transport and quantum wave interference effects, in addition to creating the entire field of mesoscopic physics 139.140 .141 .142 .143 . Although they have yet not generated a real breakthrough in microelectronics, quoting a sarcastic statement from the mainstream silicon community, "heterostructure is and will be the material of the future", they provide a foundation and rich source of inspiration for go- ing beyond the limits of conventional devices through quantum engineering of physical states in confined systems ${ }^{144.145 .146 .147 .148}$. Recently they are also subjects of rejuvenated interest as MOSFET moves toward the sub$10 \mathrm{~nm}$ era based on advanced silicon-on-insulator (SOI) structures and $S i-S i G e$ heterostructures ${ }^{149}$.

Molecules are intrinsically heterostructures. Molecular electronics offer the ultimate testing ground for quantum-effect devices based on the atom-engineering approach to the heterostructure concept. Research in this field is intimately connected to exploiting molecular electronics as artificial laboratory of new principles of nanoscopic physics 54.150 . This is still a vaguely defined area and much fundamental knowledge needs to be sorted out. But molecular heterostructures already offer multiple device opportunities that are beyond the capability of or at least very difficult to achieve in scaled silicon devices. In the case of Q1D nanostructures, this includes the possibility of fabricating metal-semiconductor and semiconductor heterojunctions with simultaneous band-gap engineering on a single nanotube and nanowire basis, and the possibility of fabricating Y-junction, T-junction, branched nanowires and superlattice devices with atomically sharp interfaces $62,63,63,83,84,151,152,153,154,155,156,157,158,159$. Similar quantum-effect devices can also be implemented on single-molecule basis through a synthetic chemistry approach, but can involve very different physical mechanisms and operation principles ${ }^{94.99}$. Some examples are single-molecule heterostructures where saturated molecular groups can be selectively inserted between molecular groups with delocalized orbitals, complex structured molecules with three-terminal or multiple-terminal configurations and charge-transfer molecular complexes. In general, electron-vibronic coupling can be strong in such single-molecule devices, whose effects need to be sorted out. The recent surge of activity on integrating molecular functionality on a semiconductor platform also brings additional functionality through contact engineering $100,121,160,161,162$ by attaching the molecule to the surface of bulk semiconductor, semiconductor quantum well, quantum wire or quantum dots.

\section{DISCUSSION AND CONCLUSION}

Central to the vision of nanotechnology is the idea that by developing and following a common intellectual path - the bottom-up paradigm of nanoscale science and technology - it will be possible in the future to assemble virtually any kind of devices or functional systems. Much thus lies in the hands of chemists and materials scientists, where the goal is to control with atomic precision the morphology, structure, composition, and size of the nanoscale building blocks. Next, understanding the physics of nanoscale materials emerging from the synthetic efforts and inserted into the device and system configurations, i.e., the effect on the operating behav- 
ior of nanostructures due to the introduction of contact, functional interface, the application of external forces and processing/environment- induced parameter variations, is a fundamental part of the bottom-up paradigm, which define properties that may ultimately be exploited for nanotechnologies and enable us to make rational predictions and define new device concepts unique to the nanoscale building blocks. Finally, to fully exploit the bottom-up paradigm, we must develop rational methods of organizing building blocks and device elements on multiple length scales. This includes not only assembling building blocks in close-packed arrays for interconnectivity but also controlling the architecture or the spacing on multiple length scales, i.e., hierarchical assembly, which must be done within the context of architectural design $62.67,68,69.70 .124,125,163$.

We have focused our attention in this work on materials and memory/logic devices. But many of the materials and device structures in molecular electronics can be easily configured for applications in chemical/bio- sensors and electromechanical devices 12.13 .55 .61 .62 .63 . In addition, molecular electronics may play an important role in solv- ing the 3-D interconnect problem in the ultimately scaled

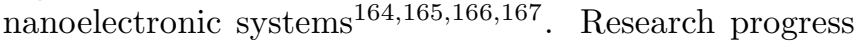
in molecular electronics systems is steady and strong, which gives us cause to believe that funtional molecular electronics systems may be practical in ten to fifteen years. Challenges to making this a reality are plentiful at every level, some naturally in the fundamental physics and chemistry of nanoelectronic materials and devices, but many in architecture and system design. These include fabricating and integrating devices, managing their power and timing, finding fault-tolerant and defect-tolerant circuits, designing and verifying billiongate systems. Any one of these could block practical molecular electronics if unsolved.

Acknowledgments We are grateful to the financial support by the DARPA MoleApps program and by the ARO/DURINT program. Y.X. has also been supported by the MARCO/DARPA Interconnect Focus Center. M.R. is also supported by the NSF Network for Computational Nanotechnology and the NASA URETI program. This paper is dedicated to Ned Seeman, visionary and friend.
* Author to whom correspondence should be addressed. E-mail: yxue@uamail.albany.edu. URL: http://www.albany.edu/ yx152122

1 Bardeen J, Brattain WH (1948) Phys Rev 74:230-231

2 Shockley W (1949) Bell Syst Tech J 28:436-489

3 Kilby, JS (1976) IEEE Trans Electron Devices ED-23:648

${ }^{4}$ Sah CT (1988) Proc IEEE 76:1280-1326

52004 International Technology Roadmap for Semiconductors. http://public.itrs.net

6 Dennar RH, Gaensslen FH, Yu HN, Ridout VL, Bassous E, LeBlanc AR (1974) IEEE J Solid-State Circuits 9:256

7 Baccarani G, Wordeman MR, Dennard RH (1984) IEEE Trans Electron Devices ED-31:452-462

8 Meindl JD, Chen Q, Davis JA (2001) Science 293:20442049

9 See, for example, Special Issue on Limits of Semiconductor Technology, Proc IEEE, Vol. 89, No. 3, 2001

10 See, for example, Special Issue on Scaling CMOS to the Limit, IBM J Res Dev, Vol. 46, No. 2/3, 2002

11 Semiconductor Research Corporation (2003) Research Needs for Novel Devices. Novel Device Task Force Report, Research Triangle Park, NC 27709

12 See, for example, Special Issue on Nanoelectronics and Nanoscale Processing, Proc IEEE, Vol. 91, No. 11, 2003

13 Luryi S, Xu J, Zaslavsky A eds (2004) Future Trend in Microelectronics: The Nano, the Giga and the Ultra. Wiley, New York

14 Semiconductor Research Corporation (2004) Silicon Nanoelectronics and Beyond: Challenges and Research Directions. Draft, Version 1.1, Research Triangle Park, NC 27709

15 http://www.nano.gov/html/facts/whatIsNano.html

16 Landauer, R (1991) Phys. Today 44:23-29

17 Leff HS, Rex AF eds (2003) Maxwell's Demon 2. Institute of Physics Publishing, Bistol Philadephia
18 Feynman R (1960) Sci Eng 23:22

19 Hey AJG, Allen RW eds (1996) Feynman Lectures on Computation. Addison-Wesley, Reading

20 Nielsen MA, Chuang IL(2000) Quantum Computation and Quantum Information. Cambridge University Press, Cambridge

21 Galindo A, Martín-Delgado (2002) Rev Mod Phys 74:347423

${ }^{22}$ Lloyd, S (2000) Nature 406:1047-1054

23 Aharonov Y, Bohm D (1961) Phys Rev 122:1649-1658

24 Aharonov Y, Bohm D (1964) Phys Rev 134:1417-1418

25 Aharonov Y, Anandan J (1990) Phys Rev Lett 65:16971700

${ }^{26}$ Margolus N, Levitin LB (1998) Physica D 120:188-195

27 Giovannetti V, LLoyd S, Maccone L (2003) Phys Rev A $67: 52109$

28 Fredkin E, Toffoli T (1982) Int J Theor Phys 21:311-325

29 Beckenstein JD (1981) Phys Rev Lett 46:623-626

30 Lloyd S, Giovannetti V, Maccone L (2004) Phys Rev Lett 93:100501

31 Bennett CH (1973) IBM J Res Dev 17:525-533

32 Landauer R (1961) IBM J Res Dev 5:183-191

33 Bennett CH (1982) Int J Theor Phys 21:905-940

34 Landauer R (1996) Science 272:1914-1918

35 Levitin LB (1998) Physica D 120:162-167

36 Meindl JD, Davis JA (2000) IEEE J Solid State Circuits 35:1515-1516

37 Keyes RW (1975) Proc IEEE 63:740-767

38 Keyes RW (2001) Proc IEEE 89:227-239

39 Keyes RW (1989) Rev Mod Phys 61:279-287

40 Keyes RW (2001) Phil Mag B 81:1315-1330

41 Plummer JD, Griffin PB (2001) Proc IEEE 89:240-258

42 Frank DJ, Dennard RH, Nowak E, Solomon PM, Taur Y, Wong HSP (2001) Proc IEEE 89:259-288

${ }^{43}$ Fischetti MV (2003) J Comp Electron 2:73-79 
44 Muller DA, Sorsch T, Moccio S, Baumann FH, EvansLutterodt K, Timp G (1999) Nature 399:758-760

45 Trumbore FA (1960) Bell Syst Tech J 39:205

46 Rousseau PM, Griffin PB, Plummer JD (1998) J Appl Phys 84:3593

47 Fahey PM, Griffin PB, Plummer JD (1989) Rev Mod Phys 61:289-384

48 Lundstrom M (1997) IEEE Electron Device Lett 18:361

49 Chang L, Choi YK, Daewon HA, Ranade P, Xiong S, Bokor J, Hu C, King TJ (2003) Proc IEEE 91:1860-1873

50 Although there is no implication on the scaling limit of the channel width from the current two-dimensional scaling models, we can reasonably expect it to be comparable to the channel length.

51 Celler GK, Cristoloveanu S (2003) J Appl Phys 93:49554978

52 Lee ML, Fitzgerald EA, Bulsara MT, Currie MT, Lochtefeld A (2005) J Appl Phys 97:11101-11128

53 Sze SM (1969) Physics of Semiconductor Devices. Wiley, New York

54 Xue Y, Ratner MA (2005) To be published

55 See, for example, the collection of articles in Molecular Electronics Special Feature, Proc Natl Acad Sci, Vol. 102, No. 26, 2005.

56 See, for example, Special Issue on Advances in Carbon Nanotubes, MRS Bulletin, Vol. 29, No. 4, 2004

57 Avouris Ph, Appenzeller J, Martel R, Wind SJ (2003) Proc IEEE 91:1772-1784

58 McEuen PL, Fuhrer MS, Park H (2002) IEEE Trans Nanotechnol 1:78-85

${ }^{59}$ Cui Y, Lieber CM (2001) Science 291:851-853

60 Cui Y, Zhong Z, Wang D, Wang WU, Lieber CM (2003) Nano Lett 3:149-152

61 Dai H (2002) Surf Sci 500:218-241

62 Lieber, CM (2003) MRS Bulletin 28:486-491

63 Yang P (2005) MRS Bulletin 30:85-91

64 Liu X, Lee R, Han J, Zhou C (2001) Appl Phys Lett 79:3329

65 Fuhrer MS, Kim BM, Duerkop, Brintlinger (2002) Nano Lett 2:755-759

66 Rosenblatt S, Lin H, Sazonova V, Tiwari S, McEuen PL (2005) Preprint

67 Huang Y, Duan X, Cui Y, Lauhon L, Kim K, Lieber CM (2001) Science 294:1313-1317

68 Melosh NA, Boukai A, Diana F, Gerardot B, Badolato A, Petroff PM, Heath JR (2003) science 112-115

69 Zhong Z, Wang D, Cui Y, Bockrath MW, Lieber CM (2003) Science 302:1377-1379

70 Jin S, Whang D, McAlpine MC, Friedman RS, Wu Y, Lieber CM (2004) Nano Lett 4:915-919

71 Javey A, Guo J, Wang Q, Lundstrom M, Dai H (2003) Nature 424:654-657

72 Javey A, Farmer DB, Guo J, Wang Q, Wang D, Gordon RG, Lundstrom M, Dai H (2004) Nano Lett 4:447-450

${ }^{73}$ Leonard F, Tersoff J (2000) Phys Rev Lett 84:4693

74 Xue Y, Ratner MA (2004) Phys Rev B 69:161402

75 Auvray S, Borghetti J, Goffman MF, Filoramo A, Derycke V, Bourgoin JP, Jost O (2004) Appl Phys Lett 84:51065108

76 Lee JU, Gipp PP, Heller CM (2004) Appl Phys Lett 85:145-147

77 Rakitin A, Papadopoulos C, Xu JM (2003) Phys Rev B $67: 33411$

78 Yao Z, Kane CL, Dekker C (2000) Phys Rev Lett 84:2941
79 Javey A, Guo J, Paulsson M, Wang Q, Mann D, Lundstrom M, Dai H (2004) Phys Rev Lett 92:106804

80 Park JY, Rosenblatt S, Yaish Y, Sazonova V, Ustunel H, Braig S, Arias TA, Brouwer PW, McEuen PL (2004) Nano Lett 4: 517

81 Perebenios V, Tersoff J, Avouris Ph (2005) Phys Rev Lett 94:86802

82 Cao J, Wang Q, Wang D, Dai H (2005) Small 1:138-141

83 Wu Y, Xiang J, Yang C, Lu W, Lieber CM (2004) Nature 430:61-65

${ }^{84} \mathrm{Wu}$ Y, Cui Y, Huynh L, Barrelet CJ, Bell DC, Lieber CM (2004) Nano Lett 4:433-436

85 Nakazato K, Blaikie RJ, Ahmed H (1994) J Appl Phys $75: 5123$

86 Tiwari S, Wahl, JA, Silva H, Rana F, Welser JJ (2000) Appl Phys A 71:403-414

87 Liu Z, Lee C, Narayanan V, Pei G, Kan EC (2002) IEEE Trans Electron Devices 49:1606

${ }^{88}$ Ganguly U, Lee C, Kan EC (2003) Mat Res Soc Symp Proc 789:N16.3

89 Ganguly U, Lee C, Kan EC (2005) Mat Res Soc Symp Proc 830:D7.5

90 Ganguly U, Kan EC, Zhang Y (2005) Appl Phys Lett $87: 43108$

91 Duan X, Huang Y, Lieber CM (2002) Nano Lett 2:487-490

92 Li C, Fan W, Lei B, Zhang D, Han S, Tang T, Lu X, Liu Z, Asano S, Meyyappan M, Han J, Zhou C (2004) Appl Phys Lett 84:1949

93 Li C, Fan W, Straus DA, Lei B, Asano S, Zhang D, Han J, Meyyappan M, Zhou C (2004) J Am Chem Soc 126:7750

94 Joachim C, Gimzewski JK, Aviram A (2000) Nature 408:541-548

95 Wada Y (2001) Proc IEEE 89:1147-1171

${ }^{96}$ Lee JO, Lientschnig G, Wiertz F, Struijk M, Janssen RAJ, Egberink R, Reinhoudt DN, Hadley P, Dekker C (2003) Nano Lett 3:113-117

97 Xu B, Xiao X, Yang X, Zang L, Tao NJ (2005) J Am Chem Soc 127:2386

98 Rosenblatt S, Yaish Y, Park J, Gore J, Sazonova V, McEuen PL (2002) Nano Lett 2:869

99 Jackel F, Watson MD, Mullen K, Rabe JR (2004) Phys Rev Lett 92:188301

100 Piva PG, DiLabio GA, Pitters JL, Zikovsky J, Rezeq M, Dogel S, Hofer WA, Wolkow RA (2005) 435:658-661

101 Heinrich AJ, Lutz CP, Gupta JA, Eigler DM (2002) Science 298:1381-1387

102 Wada Y, Uda T, Lutwyche MI, Kondo S, Heike S (1993) J Appl Phys 74:7321

103 Allmen P, Hess K (1995) Phys Rev B 52:5243

104 Lang ND (1998) Superlatt Microstruct 23:731

105 Xie FQ, Nittler, Obermair Ch, Schimmel Th (2004) Phys Rev Lett 93:128303

106 Joachim C, Gimzewski JK, Tang H (1998) Phys Rev B $58: 16407$

107 Moresco F, Meyer G, Rieder K-H, Tang H, Gourdon A, Joachim C (2001) Phys Rev Lett 86:672

108 Loppacher Ch., Guggisberg M, Pfeiffer O, Meyer E, Bammerlin M, Luthi R, Schlitter R, Gimzewski JK, Tang H, Joachim C (2003) Phys Rev Lett 90:66107

109 Chen J, Reed MA, Rawlett, Tour JM (1999) Science 286:1550

110 Collier CP, Wong EW, Belohradsky M, Raymo FM, Stoddart JF, Kuekes PJ, Williams RS, Heath JR (1999) Science 285:391 
111 Cui XD, Primak A, Zarate X, Tomfohr J, Sankey OF, Moore AL, Moore TA, Gust D, Harris G, Lindsay SM (2001) Science 294:571-574

112 Collier CP, Matterstreig G, Wong EW, Luo Y, Beverly K, Sampaio J, Raymo FM, Stoddart JF, Heath JR (2000) Science 289:1172

113 Reed MA, Chen J, Rawlett AM, Price DW, Tour JM (2001) Appl Phys Lett 78:3735

114 Li C, Zhang D, Liu X, Han S, Tang T, Zhou C, Fan W, Koehne J, Han J, Meyyappan M, Rawlett AM, Price DW, Tour JM (2003) Appl Phys Lett 82:645

115 Donhauser ZJ, Mantooth BA, Kelly KF, Bumm LA, Monnell JD, Stapleton JJ, Price DW, Rawlett AM, Allara DL, Tour JM, Weiss PS (2001) Science 292:2303

116 Ramachandran GK, Hopson TJ, Rawlett AM, Nagahara LA, Primak A, Lindsay SM (2003) Science 300:1413

117 Diehl MR, Steuerman DW, Tseng HR, Vignon SA, Star A, Celestre PC, Stoddart JF, Heath JR (2003) Chem Phys Chem 4:1335-1339

118 Kornilovitch PE, Bratkovsky AM, Williams RS (2002) Phys Rev B 66:245413

119 Troisi A, Ratner MA (2004) Nano Lett 4:591-595

120 Le JD, He Y, Hoye TR, Mead CC, Kiehl RA (2003) Appl Phys Lett 83:5518

121 Guisinger NP, Greene ME, Basu R, Baluch AS, Hersam MC (2004) Nano Lett 4:55-59

122 Sze SM (1981) Physics of Semiconductor Devices. 2nd edition. Wiley, New York

123 Mazumder P, Kulkarni S, Bhattacharya M, Sun JP, Haddard GI (1998) Proc IEEE 86:664-686

124 Heath JR, Kuekes PJ, Snider GS, Williams RS (1998) Science 280:1716-1721

125 Butts M, Dehon A, Goldstein SC (2002) Proceedings of the International Conference on Computer-Aided Design, pp 433-440

126 Von Neumann J (1966) Theory of Self-Reproducing Automata. Univerity of Illinois Press, Urbana

127 Grabert H, Devoret MH eds (1992) Single Charge Tunneling. Plenum, New York

128 Likharev KK (1999) Proc IEEE 87:606-632

129 Likharev KK (2003) Electronics Below $10 \mathrm{~nm}$. In: Greer J (eds) Nano and Giga Challenges in Microelectronics. Elsevier, Amsterdam

130 Postma HW, Teepen T, Yao Z, Grifoni M, Dekker C (2001) Scence 293:76-79

131 Woodside MT, McEuen PL (2002) Science 296:1098-1101

132 Petta JR, Salinas DG, Ralph DC (2000) Appl Phys Lett 77:4419

133 Park H, Park J, Lim AKL, Anderson EH, Alivisatos AP, McEuen PL (2000), Nature 407:57-60

134 Park J, Pasupathy AN, Goldsmith JI, Chang C, Yaish Y, Petta JR, Rinkoski M, Sethna JP, Abruna HD, McEuen PL, Ralph DC (2002) Nature 417:722-725

135 Liang W, Shores MP, Bockrath M, Long JR, Park H (2002) Nature 417:725-729

$136 \mathrm{Yu} \mathrm{H}$, Luo Y, Beverly K, Stoddart JF, Tseng HR, Heath
JR (2003) Angew Chem Int Ed 42:5706-5711

137 Pasupathy AN, Park J, Chang C, Soldatov AV, Lebedkin S, Bialczak RC, Grose JE, Donev LAK, Sethna JP, Ralph DC, McEuen PL (2005) Nano Lett 5:203-207

138 Xue Y, Ratner MA (2003) Phys Rev B 68:235410

139 Capasso F eds (1990) Physics of Quantum Electron Devices. Springer, Berlin

140 Kiehl RA, Gerhard Sollner TCL eds (1994) High Speed Heterostructure Devices. Academic Press, San Diego

141 Weisbuch C (1996) Phys Scripta T68:102-112

142 Konig U (1996) Phys Scripta T68:90-101

143 Datta S (1995) Electronic Transport in Mesoscopic Systems. Cambridge University Press, Cambridge

144 Song AM, Lorke A, Kriele A, Kotthaus JP, Wegscheider, Bichler M (1997) Phys Rev Lett 80:3831

145 Fleischmann R, Geisel T (2002) Phys Rev Lett 89:16804

146 Worschech L, Reitzenstein S, Hartmann P, Kaiser S, Kamp M, Forchel A (2003) Appl Phy Lett 83:2462

147 de Hann S, Lorke A, Kotthaus JP, Wegscheider, Bichler M (2004) Phys Rev Lett 92:56806

148 Hackens B, Gence L, Gustin C, Wallart X, Bollaert S, Cappy A, Bayot V (2004) Appl Phys Lett 4508

149 Luryi S, Zaslavsky A (2004) Solid-St Electron 48:877-885

150 Xue Y, Ratner MA (2005) Int J Quantum Chem 102:911924

151 Xu J (2003) Proc IEEE 91:1819-1829

152 Wang D, Qian F, Yang C, Zhong Z, Lieber CM (2004) Nano Lett 4:871-875

153 Menon M, Srivastava D (1997) Phys Rev Lett 79:44534456

154 Papadopoulos C, Rakitin A, Li J, Vedeneev AS, Xu JM (2000) Phys Rev Lett 85:3476-3479

155 Terrones M, Banhart F, Grobert F, Charlier JC, Terrones H, Ajayan PM (2002) Phys Rev Lett 89:075505

156 Menon M, Andriotis AN, Srivastava D, Ponomareva I, Chernozatonskii LA (2003) Phys Rev Lett 91:145501

157 Tzolov M, Chang B, Yin A, Straus D, Xu JM, Brown G (2004) Phys Rev Lett 92:75505

158 Chiu PW, Kaempgen M, Roth S (2004) Phys Rev Lett 92:246802

159 Doorn SK, O'Connell MK, Zheng L, Zhu YT, Huang S, Liu J (2005) Phys Rev Lett 94:16802

160 Briggs GAD, Fish AJ (1999) Surf Sci Rep 33:1-81

161 Wolkow RA (2000) Annu Rev Phys Chem 50:413-441

162 Hasegawaa S, Tonga X, Takedaa S, Satoa N, Nagaoa T (1999) Prog Surf Sci 60:89-257

163 Dehon A, Wilson MJ (2004) Proceedings of the International Symposium on Filed Programmable Gate Arrays, pp 123-132

164 Meindl JD, Davis JA, Zarkesh-Ha P, Patel CS, Martin KP, Kohl PA (2002) IBM J Res Dev 46:245

165 Naeemi A, Sarvari R, Meindl JD (2005) Private communications

166 Seeman NC (1998) Annu Rev Bioph Biom 27:225-248

167 Seeman NC (2004) Sci American 290:64-75 